\title{
Brief Investigation on Square Root of a Node of $T_{3}$ Tree
}

\author{
Guihong Chen"1,2, Jianhui Li1,3 \\ ${ }^{1}$ Department of Computer Science and Technology, Neusoft Institute Guangdong, Foshan, China \\ ${ }^{2}$ School of Electronics and Information Technology, Sun Yat-sen University, Guangzhou, China \\ ${ }^{3}$ State Key Laboratory of Mathematical Engineering and Advanced Computing, Wuxi, China \\ Email: chenguihong@nuit.edu.cn
}

How to cite this paper: Chen, G.H. and Li, J.H. (2018) Brief Investigation on Square Root of a Node of $T_{3}$ Tree. Advances in Pure Mathematics, 8, 666-671. https://doi.org/10.4236/apm.2018.87039

Received: June 22, 2018

Accepted: July 17, 2018

Published: July 20, 2018

Copyright (C) 2018 by authors and Scientific Research Publishing Inc. This work is licensed under the Creative Commons Attribution International License (CC BY 4.0).

http://creativecommons.org/licenses/by/4.0/

\begin{abstract}
The article investigates some properties of square root of $T_{3}$ tree's nodes. It first proves several inequalities that are helpful to estimate the square root of a node, and then proves several theorems to describe the distribution of the square root of the nodes on $T_{3}$ tree.
\end{abstract}

\section{Keywords}

Square Root, Node, Valuated Binary Tree, Inequality

\section{Introduction}

Article [1] introduced the $T_{3}$ tree and showed a number of properties of tree, including divisibility, multiples and divisors and multiplications of the nodes. Looking through the other papers that are related with the article [1], such as articles [2]-[7], one can see that the $T_{3}$ tree is really a new attempt to study integers. However, one can also see that, there has not been an article that concerns the square root of a node in the $T_{3}$ tree. As is known, a divisor of integer $N$ must be no bigger than $\sqrt{N}$. Hence the location where $\sqrt{N}$ lies in the $T_{3}$ tree is important for finding $N$ s divisor. Accordingly, this article makes an investigation on the issue and presents the results.

\section{Preliminaries}

\subsection{Symbols and Notations}

Symbol $T_{3}$ is the $T_{3}$ tree that was introduced in [1] and [2] and symbol $N_{(k, j)}$ is by default the node at position $j$ on level $k$ of $T_{3}$, where $k \geq 0$ and $0 \leq j \leq 2^{k}-1$. An integer $X$ is said to be clamped on level $k$ of $T_{3}$ if $2^{k+1}+1 \leq X \leq 2^{k+2}-1$ and 
symbol $X \hat{=} k$ indicates $X$ is clamped on level $k$. An odd interval $[a, b]$ is a set of consecutive odd numbers that take $a$ as lower bound and $b$ as upper bound, for example, $[3,11]=\{3,5,7,9,11\}$. Intervals in this whole article are by default the odd ones unless particularly mentioned. Symbol $\lfloor x\rfloor$ is the floor function, an integer function of real number $x$ that satisfies inequality $x-1<\lfloor x\rfloor \leq x$, or equivalently $\lfloor x\rfloor \leq x<\lfloor x\rfloor+1$. Symbol $A \Rightarrow B$ means conclusion $B$ can be derived from condition $A$.

\subsection{Lemmas}

Lemma 1 (See in [1]). Let $N_{(k, j)}$ be the node at the $f^{\text {th }}$ position on the $k^{\text {th }}$ level of $\boldsymbol{T}_{3}$ with $k \geq 0$ and $0 \leq j \leq 2^{k}-1$; then $2^{k+1}+1 \leq N_{(k, j)} \leq 2^{k+2}-1$.

Lemma 2 (See in [8]). For real numbers $x$ and $y$, it holds

(P2) $\lfloor x\rfloor-\lfloor y\rfloor-1 \leq\lfloor x-y\rfloor \leq\lfloor x\rfloor-\lfloor y\rfloor<\lfloor x\rfloor-\lfloor y\rfloor+1$

(P8) $n\lfloor x\rfloor \leq\lfloor n x\rfloor$ with $n$ being a positive integer

(P13) $x \leq y \Rightarrow\lfloor x\rfloor \leq\lfloor y\rfloor$

(P17) $\lfloor x\rfloor+\left\lfloor x+\frac{1}{2}\right\rfloor=\lfloor 2 x\rfloor,\left\lfloor\frac{x}{2}\right\rfloor+\left\lfloor\frac{x+1}{2}\right\rfloor=\lfloor x\rfloor$

\section{Main Results and Proofs}

Theorem 1. Let $a>1$ be an integer and $x>0$ be a real number; then it holds

$$
a^{\lfloor x\rfloor-1}<a^{\lfloor x\rfloor} \leq a^{x}<a^{\lfloor x\rfloor+1}
$$

and

$$
a^{\lfloor x\rfloor-1}<a^{\lfloor x\rfloor} \leq\left\lfloor a^{x}\right\rfloor \leq a^{\lfloor x\rfloor+1}
$$

Proof. Since $a>1$, the definition $x-1<\lfloor x\rfloor \leq x<\lfloor x\rfloor+1$ immediately yields

$$
a^{\lfloor x\rfloor-1}<a^{\lfloor x\rfloor} \leq a^{x}<a^{\lfloor x\rfloor+1}
$$

Since $a$ and $\lfloor x\rfloor$ are integers, it yields by Lemma 2 (P13)

$$
a^{\lfloor x\rfloor} \leq\left\lfloor a^{x}\right\rfloor \leq a^{\lfloor x\rfloor+1}
$$

Considering $\frac{a^{\lfloor x\rfloor}}{a^{\lfloor x\rfloor-1}}=a>1$, it knows $a^{\lfloor x\rfloor-1}<a^{\lfloor x\rfloor}$; consequently

$$
a^{\lfloor x\rfloor-1}<a^{\lfloor x\rfloor} \leq\left\lfloor a^{x}\right\rfloor \leq a^{\lfloor x\rfloor+1}
$$

Theorem 1. Let $n$ be a positive integer and

$$
b_{0}=n^{2}, b_{1}=n^{2}+1, \cdots, b_{i}=n^{2}+i, \cdots, b_{2 n}=n^{2}+2 n, b_{2 n+1}=n^{2}+2 n+1
$$

then

$$
\left\lfloor\sqrt{b_{i}}\right\rfloor_{(i=1, \cdots, 2 n)}=\left\lfloor\sqrt{b_{0}}\right\rfloor=n,\left\lfloor\sqrt{b_{2 n+1}}\right\rfloor=n+1
$$

Proof. $\left\lfloor\sqrt{b_{0}}\right\rfloor=n$ and $\left\lfloor\sqrt{b_{2 n+1}}\right\rfloor=n+1$ obviously hold. Now consider that, 
for $i=1,2, \cdots, 2 n$, it holds

$$
n=\sqrt{b_{0}}<\sqrt{b_{i}}=n \sqrt{1+\frac{i}{n^{2}}}<\sqrt{b_{2 n+1}}=n+1
$$

This is to say that, $\left.\sqrt{b_{i}}\right|_{(i=1,2, \cdots, 2 n)} \in(n, n+1)$; since $n$ is an integer, it is sure by definition of the floor function

$$
\left\lfloor\sqrt{b_{i}}\right\rfloor_{(i=1, \cdots, 2 n)}=n
$$

Corollary 1. Let $n$ and $\alpha$ be a positive integers and

$$
b_{0}=n^{2 \alpha}, b_{1}=n^{2 \alpha}+1, \cdots, b_{i}=n^{2 \alpha}+i, \cdots, b_{2 n^{\alpha}}=n^{2 \alpha}+2 n^{\alpha}, b_{2 n^{\alpha}+1}=n^{2 \alpha}+2 n^{\alpha}+1
$$

then

$$
\left.\sqrt{b_{i}}\right|_{\left(i=0,1,2, \cdots, 2 n^{\alpha}\right)}=n^{\alpha}, \sqrt{b_{2 n^{\alpha}+1}}=n^{\alpha}+1
$$

\section{Proof. (Omitted)}

Example 1. Take $b_{0}=2^{8}, b_{1}=2^{8}+1=257, \cdots, b_{2 \times 2^{4}}=2^{8}+2 \times 2^{4}=288$, $b_{2 \times 2^{4}+1}=2^{8}+2 \times 2^{4}+1=289$; then $\left\lfloor\sqrt{b_{0}}\right\rfloor=2^{4},\left\lfloor\sqrt{b_{1}}\right\rfloor=16, \cdots,\left\lfloor\sqrt{b_{2 \times 2^{4}}}\right\rfloor=16,\left\lfloor\sqrt{b_{2 \times 2^{4}+1}}\right\rfloor=17$.

Theorem 2. Let $n$ and $\alpha$ be a positive integers and

$$
\begin{aligned}
& b_{0}=n^{2 \alpha+1}, b_{1}=n^{2 \alpha+1}+1, \cdots, b_{i}=n^{2 \alpha+1}+i, \cdots, b_{2 n^{\alpha}}=n^{2 \alpha+1}+2 n^{\alpha} \sqrt{n}, \\
& b_{2 n^{\alpha}+1}=n^{2 \alpha+1}+2 n^{\alpha} \sqrt{n}+1
\end{aligned}
$$

then

$$
\left\lfloor n^{\alpha} \sqrt{n}\right\rfloor \leq\left\lfloor\sqrt{b_{i}}\right\rfloor_{\left(i=0,1,2, \cdots, 2 n^{\alpha}+1\right)} \leq\left\lfloor n^{\alpha} \sqrt{n}\right\rfloor+1
$$

Proof. See the following deductions.

1)

$$
\begin{aligned}
& b_{2 n^{\alpha}+1}=n^{2 \alpha+1}+2 n^{\alpha} \sqrt{n}+1=\left(n^{\alpha} \sqrt{n}+1\right)^{2} \Rightarrow \sqrt{b_{2 n^{\alpha}+1}}=n^{\alpha} \sqrt{n}+1 \\
& \Rightarrow\left\lfloor\sqrt{b_{2 n^{\alpha}+1}}\right\rfloor=\left\lfloor n^{\alpha} \sqrt{n}\right\rfloor+1
\end{aligned}
$$

2) By Lemma 2(P13)

$$
\begin{aligned}
& n^{2 \alpha+1}=b_{0}<b_{1}=n^{2 \alpha+1}+1<\cdots<b_{2 n^{\alpha}}=n^{2 \alpha+1}+2 n^{\alpha} \sqrt{n}<b_{2 n^{\alpha}+1}=\left(n^{\alpha} \sqrt{n}+1\right)^{2} \\
& \Rightarrow n^{\alpha} \sqrt{n}=\sqrt{b_{0}}<\left.\sqrt{b_{i}}\right|_{\left(i=1,2, \cdots, 2 n^{\alpha}\right)}<n^{\alpha} \sqrt{n}+1 \\
& \left.\Rightarrow \mid n^{\alpha} \sqrt{n}\right\rfloor=\sqrt{b_{0}} \leq\left.\sqrt{b_{i}}\right|_{\left(i=1,2, \cdots, 2 n^{\alpha}\right)} \leq\left\lfloor n^{\alpha} \sqrt{n}\right\rfloor+1
\end{aligned}
$$

Example 2. Take $b_{0}, b_{1}, \cdots, b_{2 n^{\alpha}}$ and $b_{2 n^{\alpha}+1}$ by

$b_{0}=2^{9}, b_{1}=2^{9}+1=513, \cdots, b_{2^{5}-1}=2^{9}+2^{5}-1=543, b_{2^{5}}=2^{9}+2^{5}=544$, 


$$
\begin{aligned}
& b_{2^{5}+1}=2^{9}+2^{5}+1=545 \text { then } \\
&\left.\qquad \mid \sqrt{b_{0}}\right\rfloor=\lfloor\sqrt{512}\rfloor=22, \\
&\left\lfloor\sqrt{b_{1}}\right\rfloor=\lfloor\sqrt{513}\rfloor=22, \\
&\left\lfloor\sqrt{b_{2}}\right\rfloor=\lfloor\sqrt{514}\rfloor=22, \\
& \cdots, \\
&\left\lfloor\sqrt{b_{2^{4}}}\right\rfloor=\left\lfloor\sqrt{2^{9}+2^{4}}\right\rfloor=\lfloor\sqrt{528}\rfloor=22, \\
&\left\lfloor\sqrt{b_{2^{4}+1}}\right\rfloor=\left\lfloor\sqrt{2^{9}+17}\right\rfloor=\lfloor\sqrt{529}\rfloor=23, \\
& \cdots, \\
&\left\lfloor\sqrt{b_{2 \times 2^{4}-1}}\right\rfloor=\lfloor\sqrt{543}\rfloor=23, \\
&\left\lfloor\sqrt{b_{2 \times 2^{4}}}\right\rfloor=\lfloor\sqrt{544}\rfloor=23, \\
&\left\lfloor\sqrt{b_{2 \times 2^{4}+1}}\right\rfloor=\lfloor\sqrt{545}\rfloor=23
\end{aligned}
$$

Theorem 4 Suppose integer $k$ satisfies $k>2$ and $N_{(k, 0)}$ be the leftmost node on level $k$ of $T_{3}$; then $\left\lfloor\sqrt{N_{(k, 0)}}\right\rfloor$ is even if $k$ is odd, whereas, it can be either odd or even if $k$ is even.

Proof. Since $N_{(k, 0)}=2^{k+1}+1$, it knows by Corollary $1\left\lfloor\sqrt{N_{(k, 0)}}\right\rfloor=2^{\frac{k+1}{2}}$ for an odd $k$. If $k$ is even, let it be $k=2 \alpha+1$; then by Theorem $2\left\lfloor\sqrt{N_{(k, 0)}}\right\rfloor=\left\lfloor 2^{\alpha} \sqrt{2}\right\rfloor$ or $\left\lfloor\sqrt{N_{(k, 0)}}\right\rfloor=\left\lfloor 2^{\alpha} \sqrt{2}\right\rfloor+1$, which indicates $\left\lfloor\sqrt{N_{(k, 0)}}\right\rfloor$ can be either odd or even.

Example 3. Taking $N_{(7,0)}, N_{(11,0)}, N_{(19,0)}, N_{(8,0)}, N_{(10,0)}$ and $N_{(16,0)}$ as examples results in the following results.

$$
\begin{aligned}
& N_{(7,0)}=2^{7+1}+1=257 \Rightarrow\left\lfloor\sqrt{N_{(7,0)}}\right\rfloor=16 \\
& N_{(11,0)}=2^{11+1}+1=4097 \Rightarrow\left\lfloor\sqrt{N_{(11,0)}}\right\rfloor=64 \\
& N_{(19,0)}=2^{19+1}+1=1048577 \Rightarrow\left\lfloor\sqrt{N_{(19,0)}}\right\rfloor=1024 \\
& N_{(8,0)}=2^{8+1}+1=513 \Rightarrow\left\lfloor\sqrt{N_{(8,0)}}\right\rfloor=22 \\
& N_{(10,0)}=2^{10+1}+1=1025 \Rightarrow\left\lfloor\sqrt{N_{(10,0)}}\right\rfloor=45 \\
& N_{(16,0)}=2^{16+1}+1=131073 \Rightarrow\left\lfloor\sqrt{N_{(16,0)}}\right\rfloor=362
\end{aligned}
$$

Theorem 5. Suppose integers $k$ and $j$ satisfy $k>2$ and $0 \leq j \leq 2^{k}-1$; let $N_{(k, j)}$ be the node at position $j$ on level $k$ of $T_{3}$; then it holds

$$
2^{\left\lfloor\frac{k+1}{2}\right\rfloor}-1<2^{\left\lfloor\frac{k+1}{2}\right\rfloor} \leq\left\lfloor\sqrt{N_{(k, j)}}\right\rfloor \leq 2^{\left\lfloor\frac{k}{2}\right\rfloor+2}<2^{\left\lfloor\frac{k}{2}\right\rfloor+2}+1
$$

Proof. Since $2^{k+1}+1 \leq N_{(k, j)} \leq 2^{k+2}-1$, it yields $2^{k+1}<N_{(k, j)}<2^{k+2}$; hence it 
holds

$$
2^{\frac{k+1}{2}}<\sqrt{N_{(k, j)}}<2^{\frac{k}{2}+1}
$$

By Lemma 2 (P13), it yields

$$
\left\lfloor 2^{\frac{k+1}{2}}\right\rfloor \leq\left\lfloor\sqrt{N_{(k, j)}}\right\rfloor \leq\left\lfloor 2^{\frac{k}{2}+1}\right\rfloor
$$

By Theorem 1, it holds

$$
2^{\left\lfloor\frac{k+1}{2}\right\rfloor} \leq 2^{\frac{k+1}{2}}
$$

and

$$
2^{\frac{k}{2}+1}<2^{\left\lfloor\frac{k}{2}\right\rfloor+2}
$$

Hence it results in

$$
2^{\left\lfloor\frac{k+1}{2}\right\rfloor}=\left\lfloor 2^{\left\lfloor\frac{k+1}{2}\right\rfloor}\right\rfloor \leq\left\lfloor 2^{\frac{k+1}{2}}\right\rfloor \leq\left\lfloor\sqrt{N_{(k, j)}}\right\rfloor \leq\left\lfloor 2^{\frac{k}{2}+1}\right\rfloor \leq\left\lfloor 2^{\left\lfloor\frac{k}{2}\right\rfloor+2}\right\rfloor=2^{\left\lfloor\frac{k}{2}\right\rfloor+2}
$$

That is

$$
2^{\left\lfloor\frac{k+1}{2}\right\rfloor} \leq\left\lfloor\sqrt{N_{(k, j)}}\right\rfloor \leq 2^{\left\lfloor\frac{k}{2}\right\rfloor+2}
$$

or equivalently

$$
2^{\left\lfloor\frac{k+1}{2}\right\rfloor}-1<\left\lfloor\sqrt{N_{(k, j)}}\right\rfloor<2^{\left\lfloor\frac{k}{2}\right\rfloor+2}+1
$$

Corollary 2. $\left\lfloor\sqrt{N_{(k, j)}}\right\rfloor$ is clamped in $T_{3}$ on level $\left\lfloor\frac{k+1}{2}\right\rfloor-1$ and or level $\left\lfloor\frac{k}{2}\right\rfloor$

Proof. Since $2^{\left\lfloor\frac{k+1}{2}\right\rfloor}-1$ the biggest node on level $\left\lfloor\frac{k+1}{2}\right\rfloor-2$ and $2^{\left\lfloor\frac{k}{2}\right\rfloor+2}+1$ is the smallest node on level $\left\lfloor\frac{k}{2}\right\rfloor+1$, it knows by (8) $\left\lfloor\sqrt{N_{(k, j)}}\right\rfloor$ may be clamped on levels from $\left\lfloor\frac{k+1}{2}\right\rfloor-1$ to $\left\lfloor\frac{k}{2}\right\rfloor$, totally $\left\lfloor\frac{k}{2}\right\rfloor-\left(\left\lfloor\frac{k+1}{2}\right\rfloor-1\right)+1$ levels.

By Lemma 2 (P2) $\left\lfloor\frac{k}{2}\right\rfloor-\left(\left\lfloor\frac{k+1}{2}\right\rfloor-1\right)+1 \geq 2+\left\lfloor\frac{k}{2}-\frac{k+1}{2}\right\rfloor=2-1=1$

By Lemma $2(\mathbf{P} 17 \& \mathbf{P} 8)$

$\left\lfloor\frac{k}{2}\right\rfloor-\left(\left\lfloor\frac{k+1}{2}\right\rfloor-1\right)+1=2+\left\lfloor\frac{k}{2}\right\rfloor-\left(\lfloor k\rfloor-\left\lfloor\frac{k}{2}\right\rfloor\right)=2+2\left\lfloor\frac{k}{2}\right\rfloor-\lfloor k\rfloor \leq 2$

Hence the corollary holds

Example 4. Taking the smallest nodes and the biggest nodes on level 7 and 
level 10 respectively, it can see that $\left\lfloor\sqrt{N_{(7, *)}}\right\rfloor$ is clamped on 2 levels, whereas $\left\lfloor\sqrt{N_{(10, *)}}\right\rfloor$ is clamped on 1 level.

$$
\begin{aligned}
& N_{(7,0)}=2^{7+1}+1=257 \Rightarrow\left\lfloor\sqrt{N_{(7,0)}}\right\rfloor=16 \bumpeq 2 \\
& N_{\left(7,2^{7}-1\right)}=2^{7+2}-1=511 \Rightarrow\left\lfloor\sqrt{N_{\left(7,2^{7}-1\right)}}\right\rfloor=22 \hat{=} 3 \\
& N_{(10,0)}=2^{11}+1=2047 \Rightarrow\left\lfloor\sqrt{N_{(10,0)}}\right\rfloor=45 \triangleq 4 \\
& N_{\left(10,2^{10}-1\right)}=2^{12}-1=4095 \Rightarrow\left\lfloor\sqrt{N_{\left(10,2^{10}-1\right)}}\right\rfloor=63 \stackrel{\wedge}{=}
\end{aligned}
$$

\section{Conclusion}

Elementary number theory shows that an integer must have a divisor smaller than the square root of the integer itself. Hence the square root is undoubtedly an important issue of an integer. Since $T_{3}$ tree is considered to be a new tool to study integers, the square root of a node is certainly helpful to know the distribution of the node's divisors. The properties proved in this article are sure to provide a know-about the square root of the nodes. We hope it will be useful in the future.

\section{Acknowledgements}

The research work is supported by the State Key Laboratory of Mathematical Engineering and Advanced Computing under Open Project Program No. 2017A01, the Youth Innovative Talents Project (Natural Science) of Education Department of Guangdong Province under grant 2016KQNCX192, 2017KQNCX230. The authors sincerely present thanks to them all.

\section{References}

[1] Wang, X.B. (2018) $\mathrm{T}_{3}$ Tree and Its Traits in Understanding Integers. Advances in Pure Mathematics, 8, 494-507. https://doi.org/10.4236/apm.2018.85028

[2] Wang, X.B. (2016) Valuated Binary Tree: A New Approach in Study of Integers. International Journal of Scientific and Innovative Mathematical Research, 4, 63-67.

[3] Wang, X.B. (2016) Amusing Properties of Odd Numbers Derived From Valuated Binary Tree. IOSR Journal of Mathematics, 12, 53-57.

[4] Wang, X.B. (2017) Genetic Traits of Odd Numbers with Applications in Factorization of Integers. Global Journal of Pure and Applied Mathematics, 13, 493-517.

[5] Wang, X.B. (2017) Strategy for Algorithm Design in Factoring RSA Numbers. IOSR Journal of Computer Engineering, 19, 1-7.

[6] Wang, X.B. (2017) Two More Symmetric Properties of Odd Numbers. IOSR Journal of Mathematics, 13, 37-40.

[7] Wang, X.B. (2018) Influence of Divisor-ratio to Distribution of Semiprime's Divisor. Journal of Mathematics Research, 10, 54-61. https://doi.org/10.5539/jmr.v10n4p54

[8] Wang, X.B. (2017) Brief Summary of Frequently-Used Properties of the Floor Function. IOSR Journal of Mathematics, 13, 46-48. 